PROCEEDINGS OF THE

AMERICAN MATHEMATICAL SOCIETY

Volume 133, Number 5, Pages 1411-1416

S 0002-9939(04)07753-6

Article electronically published on October 18, 2004

\title{
LEHMER'S PROBLEM FOR COMPACT ABELIAN GROUPS
}

\author{
DOUGLAS LIND
}

(Communicated by Wen-Ching Winnie Li)

\begin{abstract}
We formulate Lehmer's Problem concerning the Mahler measure of polynomials for general compact abelian groups, introducing a Lehmer constant for each such group. We show that all nontrivial connected compact groups have the same Lehmer constant and conjecture the value of the Lehmer constant for finite cyclic groups. We also show that if a group has infinitely many connected components, then its Lehmer constant vanishes.
\end{abstract}

\section{INTRODUCTION}

Let $f \in \mathbb{Z}\left[x^{ \pm 1}\right]$ be a Laurent polynomial with integer coefficients. Define its logarithmic Mahler measure to be

$$
\mathrm{m}(f)=\int_{0}^{1} \log \left|f\left(e^{2 \pi i s}\right)\right| d s
$$

Lehmer's Problem asks whether $\mathrm{m}(f)$ can be arbitrarily small but positive. Equivalently, does

$$
\inf \left\{\mathrm{m}(f): f \in \mathbb{Z}\left[x^{ \pm 1}\right], \mathrm{m}(f)>0\right\}
$$

equal zero? The smallest positive value of $\mathrm{m}(f)$ known, found by Lehmer himself [6], is attained by

$$
f_{L}(x)=x^{10}+x^{9}-x^{7}-x^{6}-x^{5}-x^{4}-x^{3}+x+1,
$$

for which $\mathrm{m}\left(f_{L}\right) \approx 0.16235$. For accounts of this problem, see [2], 3], and for connections with dynamics, see [4.

Lehmer's Problem can be formulated for arbitrary compact abelian groups. To do so, let $G$ be a compact abelian group with normalized Haar measure $\mu$. Let $\widehat{G}$ denote its (multiplicative) dual group of characters, and let $\mathbb{Z}[\widehat{G}]$ be the ring of integral combinations of characters. For $f \in \mathbb{Z}[\widehat{G}]$ define its logarithmic Mahler measure over $G$ to be

$$
\mathrm{m}(f)=\mathrm{m}_{G}(f)=\int_{G} \log |f| d \mu .
$$

Since $\log 0=-\infty$, if $f$ vanishes on a set of positive $\mu$-measure, then $\mathrm{m}(f)=-\infty$, but otherwise $\mathrm{m}(f) \geqslant 0$ by Lemma 2.1 below.

Received by the editors September 12, 2003 and, in revised form, January 7, 2004.

2000 Mathematics Subject Classification. Primary 43A40, 22D40; Secondary 37B40, 11G50.

Key words and phrases. Lehmer's Problem, Mahler measure, compact abelian group.

(C)2004 American Mathematical Society Reverts to public domain 28 years from publication 
Definition 1.1. The Lehmer constant of a compact abelian group $G$ is

$$
\lambda(G)=\inf \left\{\mathrm{m}_{G}(f): f \in \mathbb{Z}[\widehat{G}], \mathrm{m}_{G}(f)>0\right\} .
$$

Lehmer's Problem therefore asks whether $\lambda(\mathbb{T})=0$, where $\mathbb{T}=\mathbb{R} / \mathbb{Z}$.

We show in Theorem 3.2 that all nontrivial connected groups have the same Lehmer constant $\lambda(\mathbb{T})$. On the other hand, Theorem 5.1 shows that if $G$ has infinitely many connected components, then $\lambda(G)=0$. We compute the Lehmer constant of some finite groups and conjecture a simple formula for cyclic groups and also for products of two-element groups. Finally, we show that if $\lambda(\mathbb{T})=0$, then the only groups which have positive Lehmer constant are finite.

\section{Preliminaries}

Let $G$ be a compact abelian group, and let $f \in \mathbb{Z}[\widehat{G}]$. If $f$ vanishes on a set of positive $\mu$-measure, then clearly $\mathrm{m}(f)=-\infty$.

Lemma 2.1. Let $f \in \mathbb{Z}[\widehat{G}]$. If $\mu(\{x: f(x)=0\})=0$, then $\mathrm{m}(f) \geqslant 0$.

Proof. The characters appearing in $f$ generate a subgroup $\Delta$ of $\widehat{G}$. Let $H=\Delta^{\perp}$ and $\pi: G \rightarrow G / H$ be the quotient map. Then $f$ is constant on cosets of $H$, and so defines $\tilde{f} \in \mathbb{Z}\left[(G / H)^{\wedge}\right]=\mathbb{Z}[\Delta]$ such that $\tilde{f} \circ \pi=f$. Clearly $\mathrm{m}_{G / H}(\tilde{f})=\mathrm{m}_{G}(f)$, and $\tilde{f}$ vanishes on a set of positive measure if and only if $f$ does. Hence we may assume that $\widehat{G}$ is finitely generated.

Let $\mathbb{Z} / n$ denote $\mathbb{Z} / n \mathbb{Z}$. Applying the structure theorem for finitely generated abelian groups to $\widehat{G}$, we see that $G$ is isomorphic to

$$
\mathbb{Z}_{/ n_{1}} \oplus \cdots \oplus \mathbb{Z}_{/ n_{r}} \oplus \mathbb{T}^{k}
$$

for suitable integers $n_{1}, \ldots, n_{r}$ and $k$. Consider $f \in \mathbb{Z}[\widehat{G}]$ as a function of $r+1$ variables $j_{1} \in \mathbb{Z}_{/ n_{1}}, \ldots, j_{r} \in \mathbb{Z}_{/ n_{r}}$, and $\mathbf{s} \in \mathbb{T}^{k}$. Since the set where $f$ vanishes is null, each function

$$
f_{j_{1} \ldots j_{r}}(\mathbf{s})=f\left(j_{1}, \ldots, j_{r}, \mathbf{s}\right)
$$

is a nonvanishing complex combination of characters on $\mathbb{T}^{k}$, and hence by 4 , Lemma 3.7] we see that $\mathrm{m}_{\mathbb{T}^{k}}\left(f_{j_{1} \ldots j_{r}}\right)>-\infty$. Thus

$$
\begin{aligned}
\mathrm{m}(f) & =\frac{1}{n_{1} \ldots n_{r}} \sum_{j_{1}=0}^{n_{1}-1} \cdots \sum_{j_{r}=0}^{n_{r}-1} \int_{\mathbb{T}^{k}} \log \left|f_{j_{1} \ldots j_{r}}(\mathbf{s})\right| d \mathbf{s} \\
& =\frac{1}{n_{1} \ldots n_{r}} \mathrm{~m}_{\mathbb{T}^{k}}(g)>-\infty
\end{aligned}
$$

where

$$
g(\mathbf{s})=\prod_{j_{1}=0}^{n_{1}-1} \cdots \prod_{j_{r}=0}^{n_{r}-1} f_{j_{1} \ldots j_{r}}(\mathbf{s})
$$

Since for each $i$ the product over $j_{i}$ covers all the $n_{i}$ th roots of unity, the coefficients of $g$ are algebraic integers fixed by all elements in the Galois group of the field they generate, and so $g \in \mathbb{Z}\left[\left(\mathbb{T}^{k}\right)^{\wedge}\right]$. Hence $\mathrm{m}_{\mathbb{T}^{k}}(g) \geqslant 0$ by [4, Lemma 3.7], showing that $\mathrm{m}(f) \geqslant 0$.

We repeatedly use the following observation.

Lemma 2.2. Let $H$ be a closed subgroup of $G$. Then $\lambda(G) \leq \lambda(G / H)$. 
Proof. Let $\pi: G \rightarrow G / H$ be the quotient map. If $f \in \mathbb{Z}\left[(G / H)^{\wedge}\right]$, then $f \circ \pi \in \mathbb{Z}[\widehat{G}]$ and $\mathrm{m}_{G}(f \circ \pi)=\mathrm{m}_{G / H}(f)$. The conclusion then follows from the definition of $\lambda$.

\section{Connected GRoups}

In this section we prove that all nontrivial connected groups have the same Lehmer constant.

Lemma 3.1. $\lambda\left(\mathbb{T}^{k}\right)=\lambda(\mathbb{T})$ for all $k \geqslant 1$.

Proof. Since $\mathbb{T} \cong \mathbb{T}^{k} / \mathbb{T}^{k-1}$, Lemma 2.2 shows that $\lambda\left(\mathbb{T}^{k}\right) \leq \lambda(\mathbb{T})$.

To prove the reverse inequality, fix $\epsilon>0$. Choose $f \in \mathbb{Z}\left[\left(\mathbb{T}^{k}\right)^{\wedge}\right]$ with

$$
0<\mathrm{m}_{\mathbb{T}^{k}}(f)<\lambda\left(\mathbb{T}^{k}\right)+\epsilon .
$$

For $\mathbf{r}=\left(r_{1}, \ldots, r_{k}\right) \in \mathbb{Z}^{k}$ define $f_{\mathbf{r}}(s)=f\left(r_{1} s, \ldots, r_{k} s\right)$, so that $f_{\mathbf{r}} \in \mathbb{Z}[\widehat{\mathbb{T}}]$. By $[5]$ we can find $\mathbf{r}$ so that

$$
\left|\mathrm{m}_{\mathbb{T}}\left(f_{\mathbf{r}}\right)-\mathrm{m}_{\mathbb{T}^{k}}(f)\right|<\min \left\{\mathrm{m}_{\mathbb{T}^{k}}(f), \epsilon\right\} .
$$

It follows that $\lambda(\mathbb{T}) \leq \lambda\left(\mathbb{T}^{k}\right)+2 \epsilon$. Since $\epsilon>0$ was arbitrary, we obtain that $\lambda(\mathbb{T}) \leq \lambda\left(\mathbb{T}^{k}\right)$.

Theorem 3.2. If $G$ is a nontrivial connected compact abelian group, then $\lambda(G)=$ $\lambda(\mathbb{T})$.

Proof. Since $G$ is nontrivial and connected, it has a quotient isomorphic to $\mathbb{T}$, and hence $\lambda(G) \leq \lambda(\mathbb{T})$ by Lemma 2.2

To prove the reverse inequality, observe that $\widehat{G}$ is torsion-free. Let $f \in \mathbb{Z}[\widehat{G}]$ with $\mathrm{m}_{G}(f)>0$. The subgroup $\Delta$ of $\widehat{G}$ generated by the characters in $f$ is therefore isomorphic to $\mathbb{Z}^{k}$ for some $k \geqslant 1$, and so $\widehat{\Delta}=G / \Delta^{\perp} \cong \mathbb{T}^{k}$. As in the proof of Lemma 2.1 $f$ induces $\widetilde{f} \in \mathbb{Z}[\Delta]$ with $\mathrm{m}_{\widehat{\Delta}}(\widetilde{f})=\mathrm{m}_{G}(f)$. By Lemma 3.1, we have that

$$
\mathrm{m}_{G}(f)=\mathrm{m}_{\widehat{\Delta}}(\widetilde{f}) \geqslant \lambda\left(\mathbb{T}^{k}\right)=\lambda(\mathbb{T}),
$$

proving that $\lambda(G) \geqslant \lambda(\mathbb{T})$.

\section{Finite groups}

In this section we consider the problem of computing the Lehmer constant of some finite abelian groups.

Trivially $\lambda\left(\mathbb{Z}_{/ 1}\right)=\log 2$.

Define characters $\chi_{k}$ on $\mathbb{Z}_{/ n}$ by $\chi_{k}(j)=e^{2 \pi i j k / n}$. Thus the character group is $\widehat{\mathbb{Z} / n}=\left\{\chi_{0}, \chi_{1}, \ldots, \chi_{n-1}\right\}$.

Example 4.1. Let $G=\mathbb{Z}_{/ 2}$. For $f=a \chi_{0}+b \chi_{1} \in \mathbb{Z}[\widehat{\mathbb{Z} / 2}]$ we have that

$$
\mathrm{m}(f)=\frac{1}{2}(\log |a+b|+\log |a-b|)=\frac{1}{2} \log \left|a^{2}-b^{2}\right| .
$$

It is easy to check that the smallest value of $\left|a^{2}-b^{2}\right|$ greater than 1 is 3 , so that $\lambda\left(\mathbb{Z}_{/ 2}\right)=\frac{1}{2} \log 3$. 
Example 4.2. Let $G=\mathbb{Z}_{/ 3}$. A simple calculation shows that

$$
\mathrm{m}\left(a \chi_{0}+b \chi_{1}+c \chi_{2}\right)=\frac{1}{3} \log \left|a^{3}+b^{3}+c^{3}-3 a b c\right| .
$$

Choosing $a=b=1$ and $c=0$ gives a value of 2 for the expression inside the absolute value, which is clearly the smallest possible value greater than 1 . Hence $\lambda\left(\mathbb{Z}_{/ 3}\right)=\frac{1}{3} \log 2$.

Example 4.3. Let $G=\mathbb{Z}_{/ 4}$. Then

$\mathrm{m}\left(a \chi_{0}+b \chi_{1}+c \chi_{2}+d \chi_{3}\right)=\frac{1}{4} \log \left|(a+b+c+d)(a-b+c-d)\left[(a-c)^{2}+(b-d)^{2}\right]\right|$.

Putting $a=b=c=1$ and $d=0$ gives $\frac{1}{4} \log 3$.

Suppose the product inside the absolute value were \pm 2 . Then the factorization would be either $2 \cdot 1 \cdot 1,1 \cdot 2 \cdot 1$ or $1 \cdot 1 \cdot 2$. The first two factorizations cannot occur since the difference of the first two factors must be even. Therefore $|a+c| \leq 2$ and $(a-c)^{2}+(b-d)^{2}=2$. A straightforward search rules out all possibilities for attaining \pm 2 . Hence $\lambda\left(\mathbb{Z}_{/ 4}\right)=\frac{1}{4} \log 3$.

There is a simple upper bound for the Lehmer constant of a finite group.

Lemma 4.4. Let $F$ be a finite abelian group with cardinality $|F| \geqslant 3$. Then

$$
\lambda(F) \leq \frac{1}{|F|} \log (|F|-1) .
$$

Proof. Let $\chi_{0} \in \widehat{F}$ be the trivial character, and let $\delta_{0}$ be the unit mass at $0 \in F$. Then

$$
g=\sum_{\chi \in \widehat{F}} \chi=|F| \delta_{0}
$$

Put $f=g-\chi_{0}$, so that

$$
f(x)= \begin{cases}|F|-1 & \text { if } x=0 \\ -1 & \text { if } x \neq 0\end{cases}
$$

Then $\mathrm{m}_{F}(f)=\frac{1}{|F|} \log (|F|-1)>0$ since $|F| \geqslant 3$, proving (4.1).

To estimate the Lehmer constant of a cyclic group, it is convenient to introduce the following arithmetical function.

Definition 4.5. For an integer $n \geqslant 2$ let $\rho(n)$ denote the smallest prime number that does not divide $n$.

It is easy to see by using the fact that $\prod_{p \leq x} p=\exp (x(1+o(x))$ (equivalent to the Prime Number Theorem) that $\rho(n) \leq \log n(1+o(1))$ as $n \rightarrow \infty$.

Theorem 4.6. For all integers $n \geqslant 2$ we have that

$$
\lambda(\mathbb{Z} / n) \leq \frac{1}{n} \log \rho(n) .
$$

Proof. Let $p=\rho(n)$, and $\Phi_{p}$ be the $p$ th cyclotomic polynomial. Put $f_{p}(j)=$ $\Phi_{p}\left(e^{2 \pi i j / n}\right)$, so that $f_{p} \in \mathbb{Z}[\widehat{\mathbb{Z} / n}]$. Then

$$
\mathrm{m}\left(f_{p}\right)=\frac{1}{n} \log \left|R\left(\Phi_{p}(x), x^{n}-1\right)\right|,
$$


where $R(\cdot, \cdot)$ denotes the resultant. Since $p$ does not divide $n$, if follows from [1] that $R\left(\Phi_{p}(x), x^{n}-1\right)=p=\rho(n)$.

Corollary 4.7. If $n$ is odd, then $\lambda\left(\mathbb{Z}_{/ n}\right)=\frac{1}{n} \log 2$.

Proof. Since $\rho(n)=2$ we see that $\lambda\left(\mathbb{Z}_{/ n}\right) \leq \frac{1}{n} \log 2$ by Theorem 4.6. An argument similar to that in the proof of Lemma 2.1 shows the reverse inequality.

Note that the upper bound in Theorem 4.6 is actually the correct value of $\lambda(\mathbb{Z} / n)$ for $n=2,3$, and 4 computed in Examples 4.14 .2 and 4.3 above. Further computational evidence suggests the following.

Conjecture 4.8. $\lambda\left(\mathbb{Z}_{/ n}\right)=\frac{1}{n} \log \rho(n)$ for all $n \geqslant 2$.

Next, consider groups of the form $\mathbb{Z}_{/ 2} \oplus \cdots \oplus \mathbb{Z}_{/ 2}=\mathbb{Z}_{/ 2}^{n}$.

Example 4.9. Let $G=\mathbb{Z}_{/ 2} \oplus \mathbb{Z}_{/ 2}$. The characters $\chi_{i j}(x, y)=\chi_{i}(x) \chi_{j}(y)$ form the dual group $\widehat{G}=\left\{\chi_{00}, \chi_{01}, \chi_{10}, \chi_{11}\right\}$. Then

$$
\mathrm{m}\left(\sum_{i, j=0}^{1} a_{i j} \chi_{i j}\right)=\frac{1}{4} \log \left|\prod_{r, s=0}^{1}\left(\sum_{i, j=0}^{1}(-1)^{i r+j s} a_{i j}\right)\right| .
$$

The expression inside the absolute value is 3 when $a_{00}=a_{01}=a_{10}=1$ and $a_{11}=0$. It cannot attain the value \pm 2 since, if so, 2 would factor into a product of four integers whose pairwise differences are all even. Hence $\lambda\left(\mathbb{Z}_{/ 2}^{2}\right)=\frac{1}{4} \log 3$.

Further numerical work suggests the following.

Conjecture 4.10. $\lambda\left(\mathbb{Z}_{/ 2}^{n}\right)=\frac{1}{2^{n}} \log \left(2^{n}-1\right)$ for all $n \geqslant 2$.

Note that this quantity is the upper bound for $\lambda(F)$ in Lemma 4.4 when $F=\mathbb{Z}_{/ 2}^{n}$.

\section{MiXeD GROUPS}

Here we consider groups $G$ that have a nontrivial connected component $G^{0}$ of the identity.

Let $\Gamma=\widehat{G}$, and denote the torsion subgroup of $\Gamma$ by $\mathrm{t}(\Gamma)$. The totally disconnected group $G / G^{0}$ then has dual group $t(\Gamma)$.

Theorem 5.1. If $G / G^{0}$ is infinite, then $\lambda(G)=0$.

Proof. Let $\Delta$ be a finite subgroup of $\mathrm{t}(\Gamma)$, and suppose that $|\Delta| \geqslant 3$. Then by Lemma 2.2.

$$
\lambda(G) \leq \lambda\left(G / \Delta^{\perp}\right)=\lambda(\widehat{\Delta}) \leq \frac{1}{|\Delta|} \log (|\Delta|-1) .
$$

If $t(\Gamma)$ is infinite, we can then find arbitrarily large finite subgroups $\Delta$, so that the bound in (5.1) can be made arbitrarily small.

Remark 5.2. If $G^{0}$ is nontrivial, then any character of infinite order that is nontrivial on $G^{0}$ gives a quotient map of $G$ to $\mathbb{T}$, and so $\lambda(G) \leq \lambda(\mathbb{T})$ by Lemma 2.2. If $\lambda(\mathbb{T})$ were 0 , this would show that only finite groups have positive Lehmer constant. 
Example 5.3. What is the value of $\lambda(\mathbb{T} \oplus \mathbb{Z} / 2)$ ? If $\lambda(\mathbb{T})$ were 0 , the previous remark shows that $\lambda(\mathbb{T} \oplus \mathbb{Z} / 2)$ would also be 0 .

On the other hand, if $\lambda(\mathbb{T})>0$, it seems likely that $\lambda(\mathbb{T} \oplus \mathbb{Z} / 2)<\lambda(\mathbb{T})$. Some evidence for this comes from the following example, kindly communicated to us by Peter Borwein.

Let

$$
f(x)=x^{12}-x^{11}+x^{10}-x^{9}-x^{6}-x^{3}+x^{2}-x+1
$$

and

$$
g(x)=x^{8}-x^{7}+x^{6}-x^{5}+x^{4} .
$$

Then $f+g$ is cyclotomic, so that $\mathrm{m}(f+g)=0$, while $\mathrm{m}(f-g) \approx 0.30082$.

Define $h$ on $\mathbb{T} \oplus \mathbb{Z}_{/ 2}$ by

$$
h(s, j)=f\left(e^{2 \pi i s}\right)+(-1)^{j} g\left(e^{2 \pi i s}\right) .
$$

Then

$$
\begin{aligned}
\mathrm{m}_{\mathbb{T} \oplus \mathbb{Z}_{/ 2}}(h) & =\frac{1}{2}[\mathrm{~m}(f+g)+\mathrm{m}(f-g)] \\
& \approx 0.15041<0.16235=\mathrm{m}_{\mathbb{T}}\left(f_{L}\right),
\end{aligned}
$$

where $f_{L}$ is the Lehmer polynomial in (1.1). Thus $\lambda(\mathbb{T} \oplus \mathbb{Z} / 2)$ is strictly less than the best current value for $\lambda(\mathbb{T})$.

\section{REFERENCES}

[1] Tom M. Apostol, Resultants of cyclotomic polynomials, Proc. Amer. Math. Soc. 24 (1970), 457-462. MR0251010 (40:4241)

[2] David W. Boyd, Speculations concerning the range of Mahler's measure, Canad. Math. Bull. 24 (1981), no. 4, 453-469. MR0644535 (83h:12002)

[3] David W. Boyd, Reciprocal polynomials having small measure II, Math. Comp. 53 (1989), 353-357, S1-S5. MR0968149 (89m:30013)

[4] Graham Everest and Thomas Ward, Heights of Polynomials and Entropy in Algebraic Dynamics, Springer, New York, 1999. MR.1700272 (2000e:11087)

[5] Wayne M. Lawton, A problem of Boyd concerning geometric means of polynomials, J. Number Theory 16 (1983), 356-362. MR0707608 (84i:10056)

[6] D. H. Lehmer, Factorization of certain cyclotomic functions, Annals of Math. 34 (1933), 461-479. MR1503118

Department of Mathematics, Box 354350, University of Washington, Seattle, WashINGTON 98195-4350

E-mail address: lind@math.washington.edu 